\title{
Video Article \\ Manufacturing Abdominal Aorta Hydrogel Tissue-Mimicking Phantoms for Ultrasound Elastography Validation
}

\author{
Doran S. Mix ${ }^{1}$, Michael C. Stoner ${ }^{1}$, Steven W. Day ${ }^{2}$, Michael S. Richards ${ }^{3}$ \\ ${ }^{1}$ Division of Vascular Surgery, University of Rochester Medical Center \\ ${ }^{2}$ Department of Biomedical Engineering, Rochester Institute of Technology \\ ${ }^{3}$ Department of Surgery, University of Rochester Medical Center
}

Correspondence to: Michael S. Richards at michael.richards@rochester.edu

URL: https://www.jove.com/video/57984

DOI: doi:10.3791/57984

Keywords: Bioengineering, Issue 139, Ultrasound Phantoms, Elastography, Aorta Model, 3D Printing, Hydrogel, Aneurysm, Simulation

Date Published: 9/19/2018

Citation: Mix, D.S., Stoner, M.C., Day, S.W., Richards, M.S. Manufacturing Abdominal Aorta Hydrogel Tissue-Mimicking Phantoms for Ultrasound Elastography Validation. J. Vis. Exp. (139), e57984, doi:10.3791/57984 (2018).

\section{Abstract}

Ultrasound (US) elastography, or elasticity imaging, is an adjunct imaging technique that utilizes sequential US images of soft tissues to measure the tissue motion and infer or quantify the underlying biomechanical characteristics. For abdominal aortic aneurysms (AAA), biomechanical properties such as changes in the tissue's elastic modulus and estimates of the tissue stress may be essential for assessing the need for the surgical intervention. Abdominal aortic aneurysms US elastography could be a useful tool to monitor AAA progression and identify changes in biomechanical properties characteristic of high-risk patients.

A preliminary goal in the development of an AAA US elastography technique is the validation of the method using a physically relevant model with known material properties. Here we present a process for the production of AAA tissue-mimicking phantoms with physically relevant geometries and spatially modulated material properties. These tissue phantoms aim to mimic the US properties, material modulus, and geometry of the abdominal aortic aneurysms. Tissue phantoms are made using a polyvinyl alcohol cryogel (PVA-c) and molded using 3D printed parts created using computer aided design (CAD) software. The modulus of the phantoms is controlled by altering the concentration of PVA-C and by changing the number of freeze-thaw cycles used to polymerize the cryogel. The AAA phantoms are connected to a hemodynamic pump, designed to deform the phantoms with the physiologic cyclic pressure and flows. Ultra sound image sequences of the deforming phantoms allowed for the spatial calculation of the pressure normalized strain and the identification of mechanical properties of the vessel wall. Representative results of the pressure normalized strain are presented.

\section{Video Link}

The video component of this article can be found at https://www.jove.com/video/57984/

\section{Introduction}

Abdominal aortic aneurysms (AAA) are focal enlargements of the aorta that occur preferentially near the aortic bifurcation ${ }^{1}$. The exact cause of AAA formation is unknown, although many theories suggest that the pathogenesis is multifactorial, with genetic, behavioral, hemodynamic, and environmental factors contributing ${ }^{2,3}$. While the diagnosis of an abdominal aortic aneurysm can be obtained using non-invasive imaging techniques, the prediction of patient-specific rupture risk is not as precise ${ }^{4,5,6}$. Surgical repair can reduce the risk of aortic rupture, but operative repair of the aorta carries a high rate of associated morbidity and mortality ${ }^{7}$. Current surgical practices use the "maximum size criteria", or maximum absolute diameter of the aneurysms, to predict a patient's risk of rupture. Unfortunately, it has been well established that an aneurysm still ruptures below sizes clinically acceptable for surgical repair, meaning that patients with any sized aneurysm carry some risk of rupture $^{8,9,10,11,12,13}$. Additionally, it is known that historical reports of rupture risk are likely over-estimations of the true rupture risk, meaning many patients are exposed to surgical risk without benefit ${ }^{13}$. A more accurate assessment of the patient-specific rupture risk is needed to help stratify a patient's risk-benefit ratio of undergoing surgical aneurysm repair.

It has been shown that the spatial stress distribution within an AAA is of critical importance in determining rupture potential and may be a better indicator than maximum diameter ${ }^{14,15,16,17,18}$. Most of the recent studies that investigate the mechanics of AAA rupture use segmented geometries from X-ray computed tomography (CT) images, and population averaged mechanical properties of aortic tissue measured ex vivo. Finite element (FE) models are then used to predict the vessel wall stresses ${ }^{14,15,16,17,18}$. However, because the mechanical properties are determined following the tissue excision, it is unclear whether the resulting models accurately depict the resulting in vivo patient-specific stresses. These studies typically assume homogeneous vessel wall material properties and don't account for the highly heterogeneous structure of the aortic wall and thrombus ${ }^{19,20,21,22,23,24,25}$

Ultrasound-based elasticity imaging is used clinically to diagnose and monitor a variety of disease pathologies ${ }^{26}$. This technology provides a non-invasive means to interrogate the physical interactions of soft tissues. Vascular US elasticity imaging has been used as an adjunct imaging modality to clinical US evaluation in the screening and monitoring of AAAs. The combination of these techniques provides both geometric 
information, such as diameter and length, as well as mechanical data, such as relative stiffness and stiffness variation. While many elasticity imaging techniques require an external load to induce a measurable tissue deformation, the tissue motion to be measured here is induced by changes in the aortic pressure caused by the beating heart. Numerous methods have been published to spatially resolve strain fields in deforming vessels, however, validation studies of these methods have been limited to human patients, animal models, or ex vivo tissue samples $^{27,28,29,30,31,32}$. To date, few methods allow for creations of custom geometries with spatially varied material properties ${ }^{27,29}$

Here we present a method of manufacturing US compatible, tissue-mimicking phantoms that can be tailored to a variety of relevant aortic geometries and material properties for validation of US elastography techniques. Although previous groups have been able to design complex geometry phantoms to mimic AAA geometries using 3D printing technology ${ }^{33,34}$, printable rubbers are known to have a high attenuation to US and do not have a means to later their material properties. Phantoms are made from polyvinyl alcohol cryogel (PVA-c), which has been previously shown to be ideal for mimicking vascular tissue properties ${ }^{35}$. These phantoms can be used in US, magnetic resonance, and elastographic imaging ${ }^{36,37,38}$. The aortic aneurysm geometry was designed similarly to that of the simulation model created by Vorp et al. ${ }^{14}$. The vessel has a nominal diameter of $22.5 \mathrm{~mm}$ and has an aneurysmal bulge that is $64 \mathrm{~mm}$ bulge long, $47 \mathrm{~mm}$ in diameter and eccentric $(\beta$ $=0.6)^{14}$ to the anterior side of the phantom. The last section mimics the iliac bifurcation with a distal diameter of $15 \mathrm{~mm}$. The phantom was chosen to have a constant thickness of approximately $5 \mathrm{~mm}$. Raghavan et al. reported in a small study that the vessel thickness of AAA ranges from $0.23-4.26 \mathrm{~mm}$, with a median value of $1.48 \mathrm{~mm}^{39}$. A nominal vessel thickness on the larger end of that spectrum was chosen here for manufacturing concerns with the expectation that improved 3D printing techniques will improve the minimum phantom thickness that is able to be molded. Phantom molds were designed in CAD and are 3D printed using commercially available printers and filament.

The molds are injection filled with the PVA-c solution and subjected to a series of freeze/thaw cycles $\left(-20^{\circ} \mathrm{C}\right.$ and $\left.+20^{\circ} \mathrm{C}\right)$ to cross-link the PVA$c$ polymer and polymerize the gel. The elastic modulus of the PVA-c is controlled by altering the concentration of the PVA-c gel or the number of freeze-thaw cycles. The aneurysmal section of the phantom required loss mold to remove from the inner lumen of the vessel. This was accomplished by the use of a polyvinyl alcohol, 3D printer filament (PVA). Although chemically similar to the PVA-c powder, the PVA filament does not polymerize when frozen and, as such, can be dissolved in water after the PVA-c has been set. Additional sample molds are printed to create tensile testing specimens, in a "dog bone" configuration, with the same PVA-c concentration. These molds undergo the same freeze/ thaw cycles and are used for tensile testing to independently measure the elastic modulus of the phantom sections. A background material was manufactured with softer PVA-c, made to simulate tissues of the retroperitoneum ${ }^{40,41}$. This background phantom was manufactured as a homogeneous axisymmetric cylindrical tube with a $4 \mathrm{~cm}$ inner diameter, a $16.5 \mathrm{~cm}$ outer diameter, and a length of $16.5 \mathrm{~cm}$. It was made from a $5 \%$ PVA solution and subjected to a total of two freeze-thaw cycles.

The final AAA phantoms were placed in the background phantom and connected, via tube fittings and clamps, to a hemodynamic water pump designed to deform the phantoms with physiologic cyclic flows and pressures. The pump speed was set to deliver approximately a 6-7 kPa pressure pulse at a rate of approximately $1 \mathrm{~Hz}$. Ultra sound image sequences of the deforming phantoms were collected, and the pressure normalized strain was calculated to identify differences in the spatially varied mechanical properties. Representative results of the pressure normalized strain images within the vessel region are presented. The increasing regional differences in the normalized strain of the stiffer heterogenous phantoms, relative to the homogeneous phantom, demonstrate the differences in the vessel stiffness and our ability to measure it.

Protocol

\section{Download STL Models from the NIH 3D Print Exchange}

1. Navigate to the NIH 3D Print Exchange (3dprint.nih.gov) and in the Search entry type Simulated Aneurysmal Aortic Phantom Mold and hit the enter key.

2. In the subsequent list returned from the search, find the model " 3DPX-009210" and click that entry.

3. Click the download button and subsequently click the Simulated Aneurysmal Aortic Phantom Mold.zip file from the drop-down list to download this file.

4. Double click the downloaded file to unzip it and store the resulting files (InnerDistSTL.stl, InnerProxSTL.stl, OuterAntSTL.stl, OuterPostSTL.stl, BackgroundMoldSTL.stl and SampleMoldSTL.stl) to the computer used for 3D printing in Steps 2.1-2.7. NOTE: One may alternatively download each of the files listed in Step 1.4 separately.

\section{3D Printing of Molds}

1. Open the 3D printer interface software and use the Connect button to connect to the printer.

2. Import the downloaded STL file OuterAntSTL.stl (Figure 1a, blue) into the 3D printing software. In the 3D printing software, select the Edit button and orient the mold part by clicking the Rotate menu and then clicking the $\mathbf{X}, \mathbf{Y}$, or $\mathbf{Z}$ buttons to align the long axis parallel to the print bed with the outside of the mold facing the print bed. Click the Save button and then click the Print button and print the mold part using polylactic acid (PLA) plastic filament on a single extruder. 

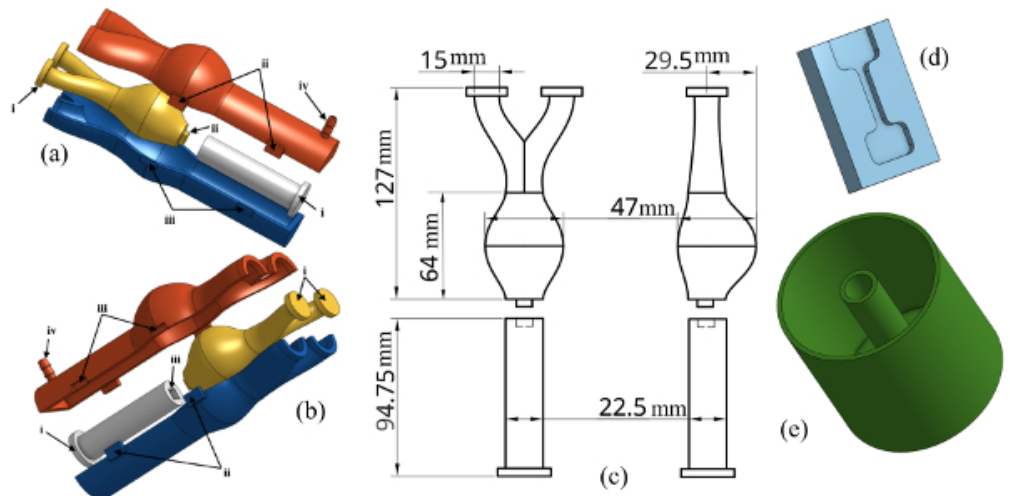

Figure 1: CAD Representation of Phantom, Background and Sample Molds. (a) - (b) 3D CAD images of vessel mold and orientation of parts for assembly. Registration spacers (i), pins (ii), holes (iii) and the fill hole are shown. (c) Drawing of inner lumen highlighting the inner vessel dimensions. (d) CAD rendering of the sample molds. (e) CAD rendering of the background phantom mold. Please click here to view a larger version of this figure.

3. Repeat Step 2.2 for the OuterPostSTL.stl file (Figure 1a, red).

4. Following the same process from Step 2.2, import the STL file InnerDistSTL.stl (Figure 1a, white) into the 3D printing software and select the "Edit" button and in the Rotate menu click the $\mathbf{X}, \mathbf{Y}$, or $\mathbf{Z}$ buttons to align the long axis perpendicular to the print bed and such that the registration pin (i) is in contact with the print bed. Click the Save button and then click the Print button and print the mold part using PLA plastic filament on a single extruder.

NOTE: Do not print this part with the support structure. Do not use more than $30 \%$ infill for this printed part.

5. Import the STL file SampleMoldSTL.stl (Figure 1d) into the 3D printing software. Select the Edit button and in the Rotate menu click the $\mathbf{X}$, $\mathbf{Y}$, or $\mathbf{Z}$ buttons to align the part such that the inside of the mold is facing up from the print bed. Click the Save button and then click the Print button and print the mold part using PLA plastic filament on a single extruder.

NOTE: Do not print this part with the support structure. Print 3 or more sample molds.

6. Import the STL file BackgroundMoldSTL.stl (Figure 1e) into the 3D printing software. Select the "Edit" button and in the Rotate menu click the $\mathbf{X}, \mathbf{Y}$, or $\mathbf{Z}$ buttons to align the part such that the bottom of the mold (i.e., the closed end of the cylinder) is facing the print bed. Click the Save button and then click the Print button and print the mold part using PLA plastic filament on a single extruder. NOTE: Do not print this part with the support structure.

7. Import the STL file InnerDistSTL.stl (Figure 1a, yellow) into the 3D printing software. Select the "Edit" button and in the Rotate menu click the $\mathbf{X}, \mathbf{Y}$, or $\mathbf{Z}$ buttons to align the part such that the long axis is perpendicular to the print bed and bifurcation registration pins (i) are facing the print bed. Click the Save button and then click the Print button and print the mold part using polyvinyl acid (PVA) plastic filament on a single extruder.

8. Remove any support material from the 3D printed parts of Steps 2.1-2.7 (Figure 2a).

NOTE: It is not necessary to remove the support structure from the outer mold parts if they do not interfere with the mold assembly. 

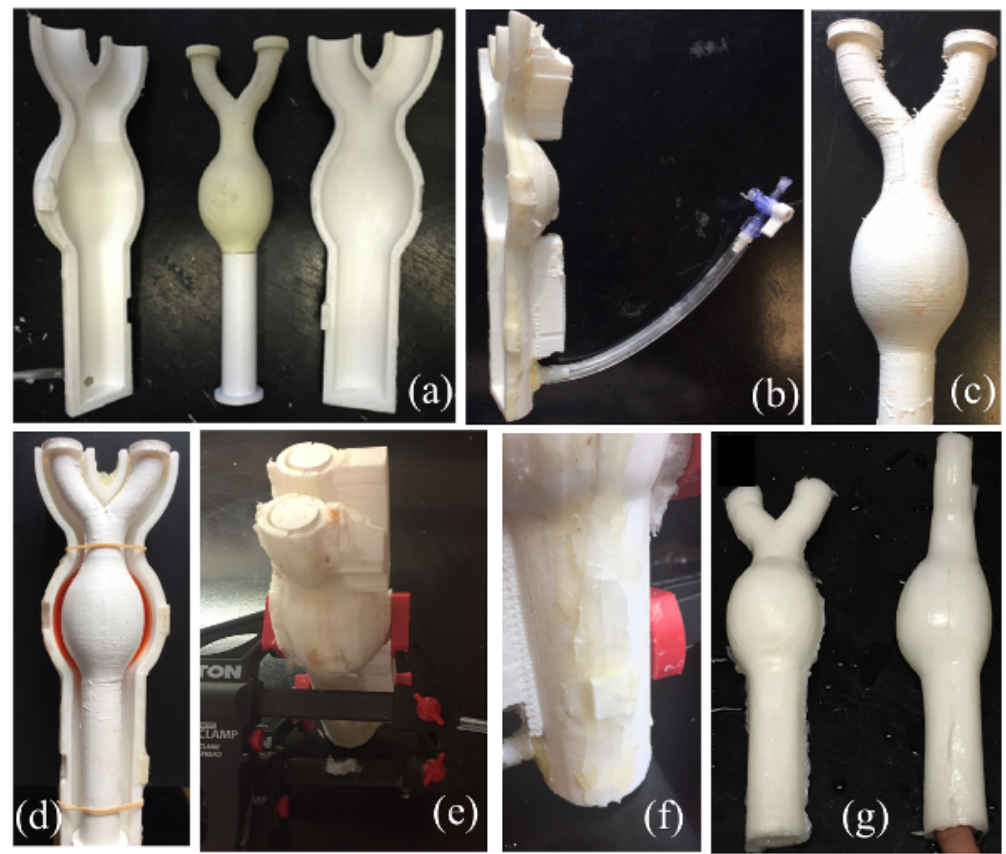

Figure 2: Vessel Phantom Mold Assembly and Final Vessel Phantom. (a) The final printed mold of inner and outer lumen molds. The distal end of the inner lumen printed in a dissolvable PVA plastic and is attached to the proximal end of the inner lumen mold using deformable wax. (b) Tubing attached to the injection port of the outer lumen mold and the syringe stopper. (c) The inner lumen mold after spray coating of flexible sealant. (d) Assembly of bulge side of the outer lumen mold and inner lumen mold with PVA-c (dyed red) added for stiff aneurysm phantoms. (e) Full vessel mold assembled and clamped. (f) Deformable wax applied to the seams of the outer lumen mold to prevent PVA-c from leaking from the mold. (g) Final PVA-c phantom after 5 freeze/thaw cycles and removal from the mold. Please click here to view a larger version of this figure.

\section{Hydrogel Preparation}

1. Mix $22.2 \mathrm{~g}$ of PVA-c powder in $200 \mathrm{~mL}$ of tap water (10\% by mass) in a glass beaker. Microwave the solution to a boil and stir. Repeat this step until all of the PVA powder is dissolved and the solution appears translucent.

2. Suspend $0.4 \mathrm{~g}$ of calcium carbonate powder $(0.2 \%$ by mass $)$ in $10 \mathrm{~mL}$ of water and add to the solution from Step 2.1 to act as ultrasound scatterers. Mix Thoroughly. Cover the solution and allow it to cool to the room temperature (RT). NOTE: For homogeneous phantoms skip to Step 3.5

3. Mix $17.6 \mathrm{~g}$ of PVA-c powder in $100 \mathrm{~mL}$ of tap water (15\% by mass or as desired) in a separate glass beaker. Microwave the solution to a boil and stir. Repeat this step until all the PVA powder is dissolved and the solution appears translucent.

4. Suspend $0.4 \mathrm{~g}$ of calcium carbonate powder $(0.2 \%$ by mass $)$ in $5 \mathrm{~mL}$ of water and add to the solution from Step 2.3. Mix Thoroughly. Cover the solution and allow it to cool to the RT.

5. Mix $183.7 \mathrm{~g}$ of PVA-c powder in $3.5 \mathrm{~L}$ of tap water (5\% by mass) in a separate large pot. Bring the solution to a boil and stir. Remove the pot from the heat once the PVA powder is dissolved and the solution appears translucent.

6. Suspend $7.4 \mathrm{~g}$ of calcium carbonate powder $(0.2 \%$ by mass $)$ in $10 \mathrm{~mL}$ of water and add to the solution from Step 2.5 . Mix Thoroughly. Cover the solution and allow it to cool to the RT.

\section{Assembly of Molds}

1. Attach approximately $100 \mathrm{~mm}$ of the flexible tubing to the injection port of the outer lumen mold. To the opposite end of the tubing, attach a stopcock with syringe connections (Figure $\mathbf{2 b}$ ).

2. Align the registration pins of the inner lumen mold and, using deformable wax, adhere the bulging vessel part of the inner lumen mold to the straight vessel part of the inner lumen mold.

3. In a well-ventilated area, apply a spray-on flexible rubber coating to the aneurysmal end of the inner lumen mold to prevent the hydrogel from dissolving the PVA mold part during the molding process (Figure 2c). NOTE: For homogeneous phantoms skip to Step 4.6.

4. With the larger side of the aneurysmal part of the outer mold facing down, fill the bulge with $15 \mathrm{~mL}$ of the solution created in Steps 3.3-3.4 (Figure 2b,). Place the assembled inner mold parts in the front outer mold part (Figure 2d). Use rubber bands to hold the inner lumen part in place.

NOTE: In Figure 2, PVA-c is dyed red for visibility.

5. Freeze the mold assembly in a $-20^{\circ} \mathrm{C}$ freezer for $12 \mathrm{~h}$ and remove from the freezer. Move on to Step 4.6 without letting the solution in the mold assembly thaw.

6. While waiting for the mold to freeze (Step 4.4), apply a generous amount of deformable wax to the Back surface of a printed sample mold and clamp it to a flat plastic sheet cut to the minimum size of approximately $100 \mathrm{~mm}$ by $60 \mathrm{~mm}$ by $10 \mathrm{~mm}$ (Figure 3a). Fill the space between the mold and the plastic sheet with the same PVA solution used in Step 4.3. Freeze the sample mold in the same freezer $\left(-20^{\circ} \mathrm{C}\right)$ as the vessel mold in Step 4.4 . 

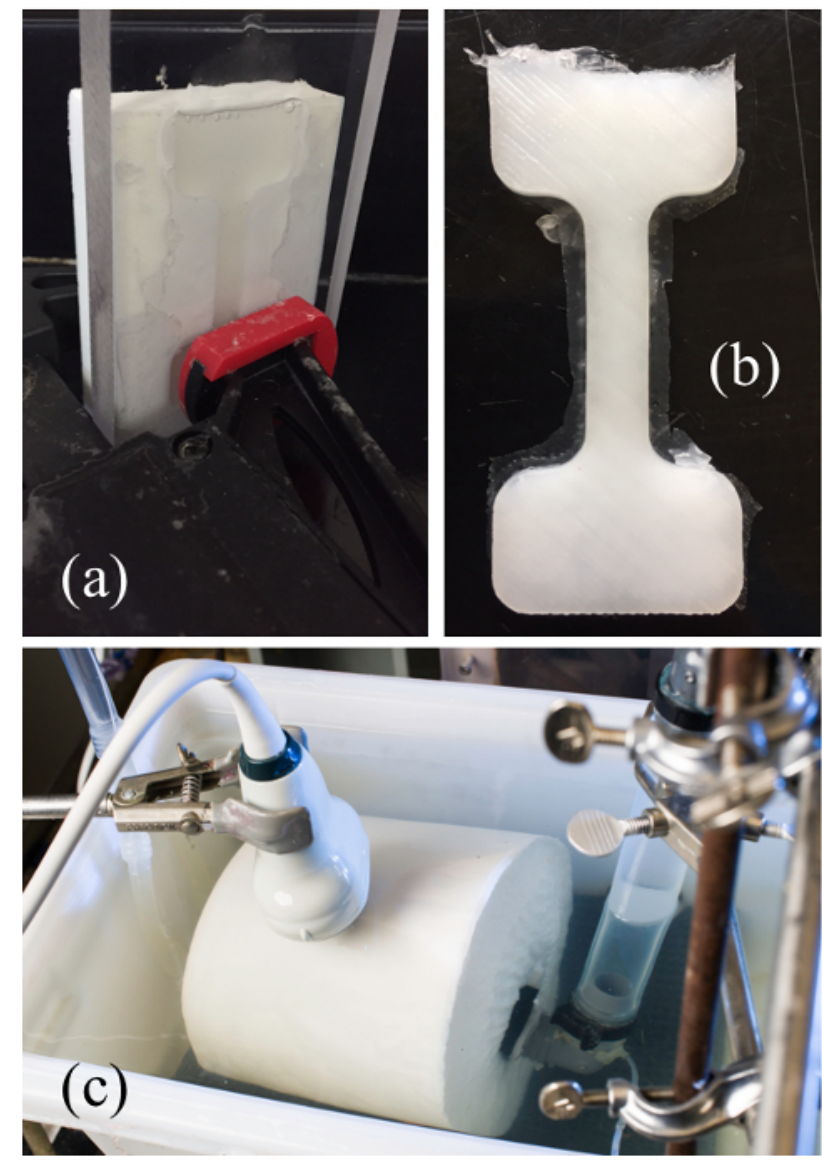

Figure 3: Sample mold and Final Sample and Background Phantoms. (a) Clamped sample mold and clear plastic sheet. PVA-c is poured into the sample mold and air bubbles are allowed to surface. (b) PVA-c sample after final freeze/thaw cycle. (c) Experimental US imaging setup of phantom attached to simulator pump and placed in the background PVA-c phantom. Please click here to view a larger version of this figure.

7. Assemble and clamp together the entire vessel mold in the orientation shown in Figure 1a and 1b (Figure 2e). Line the seams of the outer lumen molds using a deformable wax to ensure that the hydrogel does not leak during injection (Figure 2f).

8. Fill a $60 \mathrm{~mL}$ syringe with the PVA-c solution made in Steps 3.1 and 3.2. With the bifurcation end of the mold up inject the PVA-c solution into the mold assembly avoiding air bubbles in the injected solution.

NOTE: If any leaks occur during the injection, pause injection and patch leaky areas with deformable wax. Repeat syringe injections until the PVA-c solution fills the mold.

9. Allow the mold to sit for $30 \mathrm{~min}$, tapping the mold gently every $10 \mathrm{~min}$ to allow any air bubbles to rise to the top of the mold. Repeat the syringe injection if needed to top off the mold. Freeze the entire mold assembly for $12 \mathrm{~h}$ and remove from the freezer. Allow the mold assembly to thaw at RT for $12 \mathrm{~h}$.

10. While waiting for the mold to freeze (Step 4.8), assemble and clamp another sample mold and flat plastic sheet cut as described in Step 4.5 (Figure 3a). Fill the space between the mold and the plastic sheet with the same PVA solution used in Step 4.7. Freeze and thaw the sample mold in the same freezer $\left(-20^{\circ} \mathrm{C}\right)$ and at the same time as the vessel mold in Step 4.8 and the sample mold of Step 4.5.

11. Freeze and thaw the vessel mold and both the sample molds from Steps $4.5,4.8$ and 4.9 four more times, for a total five 24 -h freeze/thaw cycles. After the $5^{\text {th }}$ freeze/thaw cycle, remove the PVA-c testing samples from their molds (Figure $3 \mathbf{b}$ ). Trim any excess cryogel from the samples and store them in a sealed container of a $5 \%$ by volume bleach/water solution at RT.

12. Remove the PVA-c vessel from the outer lumen mold. Carefully separate the straight vessel part of the inner lumen mold from the aneurysmal part and remove from the PVA-c vessel. Cut the registration spacers from the bifurcated end of the aneurysmal part of the inner lumen mold to expose the printed PVA filament. Place in a water bath at RT to dissolve the PVA aneurysmal part. NOTE: This may take $24 \mathrm{~h}$ or more, however, adding warm water to the bath may speed the dissolving process.

13. After dissolving and removing the PVA printed part from inside of the vessel phantom, store the phantom in a sealed container of a $5 \%$ by volume bleach/water solution at RT.

14. Fill the background mold with approximately $3.3 \mathrm{~L}$ of the PVA-c solution made in Steps 3.5 and 3.6 . Freeze $\left(-20^{\circ} \mathrm{C}\right)$ the background mold for $12 \mathrm{~h}$ and remove from the freezer. Allow the mold to thaw at RT for $12 \mathrm{~h}$ and repeat for a total of 2 freeze/thaw cycles.

15. At the same time as Step 4.13, fill a sample mold assembly with the same PVA-c solution used in Step 4.13 and put it through the same freeze/thaw samples as the background mold.

16. After the $2^{\text {nd }}$ thaw, remove the background sample and background phantom from their molds and store them in a sealed container of a $5 \%$ by volume bleach/water solution at RT. 


\section{Phantom and Sample Testing}

1. Place the vessel phantom and background phantom into a large water bath. Attach the larger vessel end to the output of the hemodynamic water pump ${ }^{42,43}$ using tubing clamps (Figure 3c). Place the vessel phantom in the background phantom and then attach the bifurcated ends of the phantom to the inlet to the hemodynamic pump using tubing clamps.

2. Place a solid-state pressure sensor catheter in the system of the vessel and pump near the inlet of the hemodynamic pump. Run the hemodynamic pump such that the pressures of the wall deformations are between a minimum of $0 \mathrm{kPa}$ and a maximum $7.5 \mathrm{kPa}(\mathrm{Figure} 4 \mathrm{a})$.

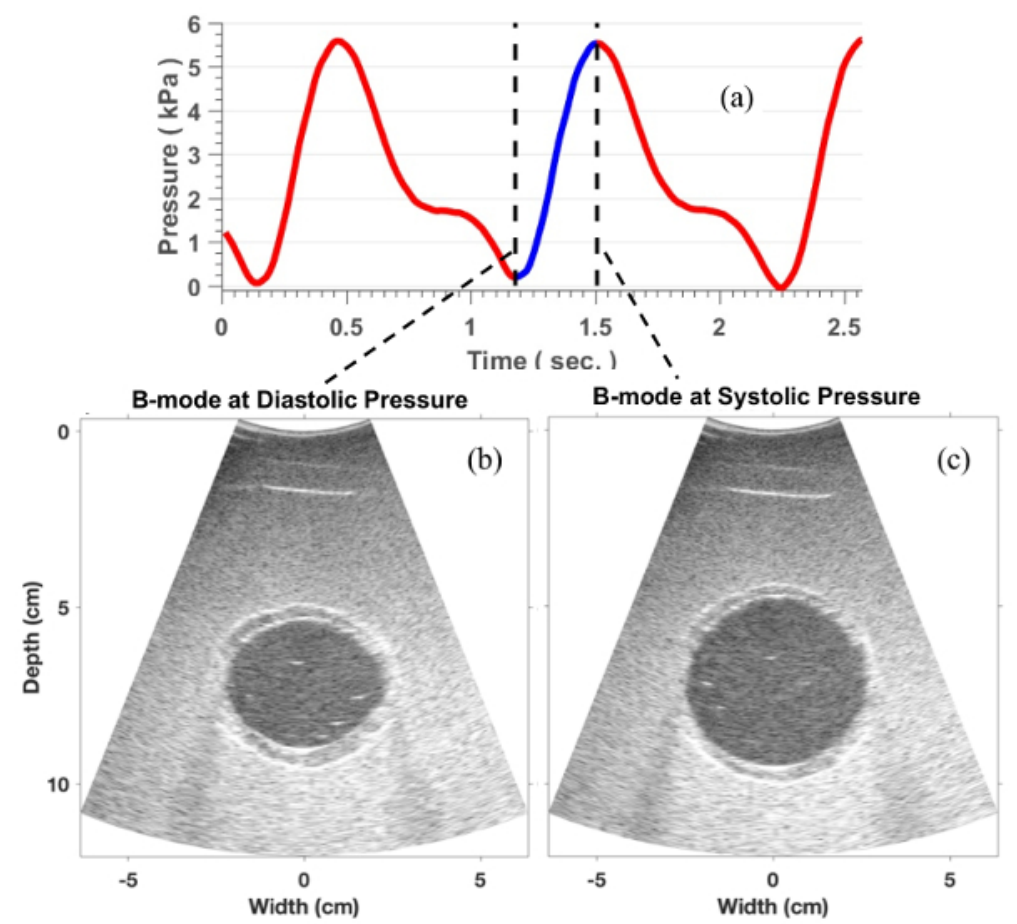

Figure 4: Imaging Protocol. (a) Pressure profile measured during the phantom imaging setup. (b) A representative B-mode image of the phantom at the minimum pressure. (c) B-mode at maximum pressure. Please click here to view a larger version of this figure.

3. Use an ultrasound (US) system and a convex transducer with a center frequency of approximately $5 \mathrm{MHz}$ to collect US Images of the background and vessel phantoms in cross-section at the location of the maximum vessel diameter (Figure $\mathbf{4 b}$ and $\mathbf{4 c}$ ). Record the pressure data using a digital acquisition system (Figure 4a).

NOTE: Details for performing the image acquisition in this step can be found in Mix et al ${ }^{44}$.

4. Obtain the displacement estimations by using a non-rigid image registration-based technique as described in Mix et al. ${ }^{44}$. From the measurements of the two-dimensional (2D) displacement field $\left(\boldsymbol{u}_{i}(\boldsymbol{x})\right)$, calculate the 2D strain tensor field $\left(\varepsilon_{i j}(\boldsymbol{x})\right)$ by evaluating the symmetric part of the gradient of the displacement field:

$\varepsilon_{i j}=\frac{1}{2}\left(\frac{\partial u_{i}}{\partial x_{j}}+\frac{\partial u_{j}}{\partial x_{i}}\right)$.

5. Then, calculate the maximum principal strain $\left(\varepsilon_{p}\right)$ as the maximum principal component of the strain tensor field using the following equation:

$\varepsilon_{p}=\frac{\varepsilon_{11}+\varepsilon_{22}}{2}+\sqrt{\left(\frac{\varepsilon_{11}-\varepsilon_{22}}{2}\right)^{2}+\left(\frac{\varepsilon_{12}}{2}\right)^{2}}$

6. Lastly, determine the frame of the principal strain at the peak pressure and divide this strain tensor field by difference in the maximum and minimum catheter measured pressures (Figure $4 a)$, or the pulse pressure (PP), to spatially resolved pressure normalized principle strain $\left(\varepsilon_{p} /\right.$ PP). 

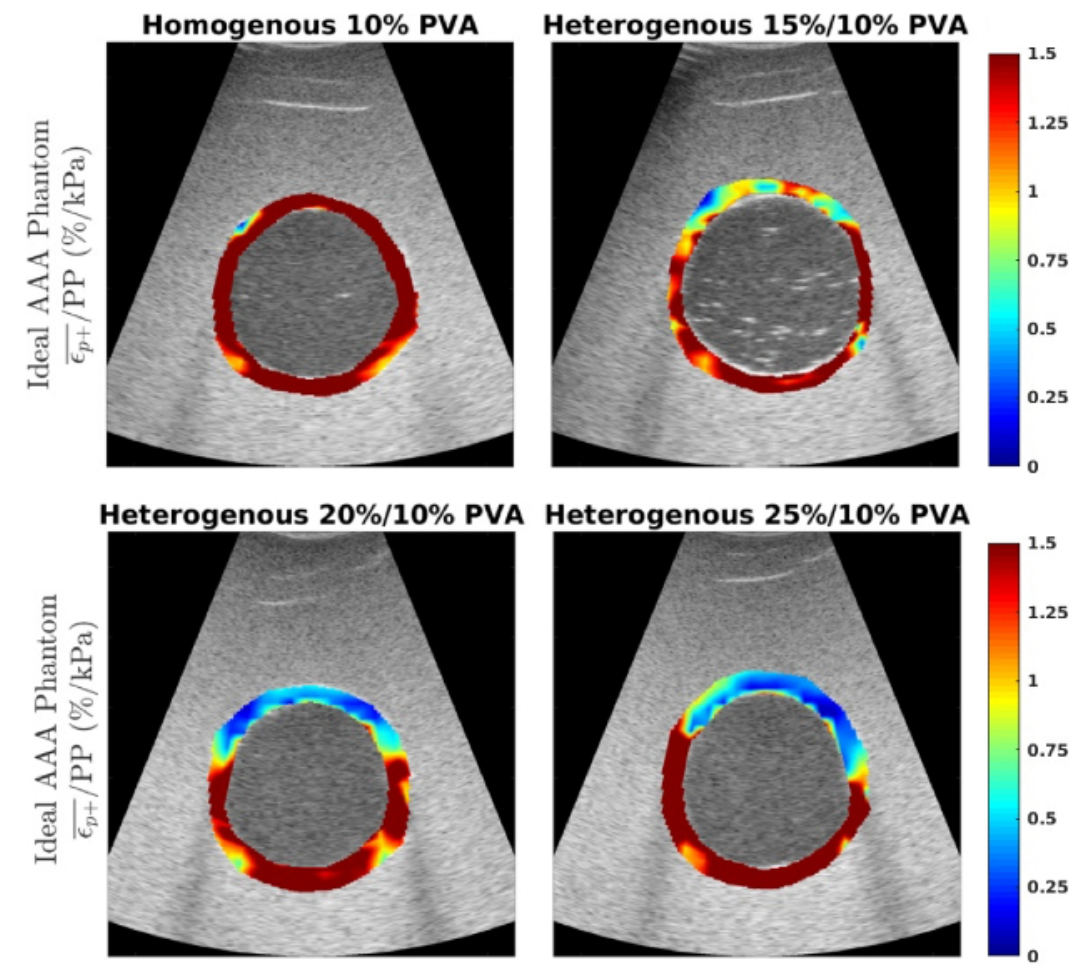

Figure 5: Pressure Normalized Strain Images. Representative images of the pressure normalized strain $\left(\varepsilon_{\mathrm{p}} / \mathrm{PP}\right)$ in $\% / \mathrm{kPa}$ measured within the vessel for the homogeneous $10 \%$ by mass vessel phantom (a) and the heterogeneous phantoms with a $15 \%$ by mass (b), $20 \%$ by mass and $25 \%$ by mass anterior aneurysmal section (top of vessel). This figure has been modified from Mix et al. ${ }^{44}$. Please click here to view a larger version of this figure.

\section{Representative Results}

Representative B-mode images of the vessel mimicking phantoms are shown for the minimum and maximum pressures measured by the catheter (Figure $\mathbf{4 b}$ and $\mathbf{4 c}$, respectively). The pressure-normalized strain $\left(\varepsilon_{\mathrm{p}} / \mathrm{PP}\right)$ in\%/kPa is shown for four different manufactured phantoms (Figure 5). Figure 5a shows the measured pressure-normalized strain within a homogeneous phantom manufactured with a $10 \%$ by mass PVA-c solution. The ratio of the average strain measured within the posterior quarter (image bottom) of the phantom to the average strain in the anterior quarter (image top) was 0.92 . Figure $\mathbf{5 b}$ shows $\varepsilon_{\mathrm{p}} / \mathrm{PP}$ for a phantom in which the aneurysmal section of the phantom was manufactured with a $15 \%$ by mass PVA-c solution and the remainder of the phantom was made using the $10 \%$ by mass PVA-c. The ratio of posterior to anterior strain for this phantom was found to be 1.87 . Figure $5 c$ shows $\varepsilon_{\mathrm{p}} / \mathrm{PP}$ for the heterogeneous phantom with $20 \%$ by mass PVA-c, with a posterior to anterior strain ratio of 4.23 . Figure $5 \mathrm{~d}$ shows $\varepsilon_{\mathrm{p}} / \mathrm{PP}$ for the heterogeneous phantom with $25 \%$ by mass PVA-c, with a posterior to anterior strain ratio of 7.37 .

The results presented here show that abdominal aortic phantoms were created with complex geometries and spatially varying material properties. Design of phantom geometries were, or more specifically, phantom molds were done using computer software which facilitates phantom geometry alterations (Figure 1a and 1b). Molds can be readily 3D printed and assembled and complex mold geometries can be printed using PVA filament and removed, similar lost-wax casting techniques. The final vessel phantoms can be dynamically pressurized and are stable under large loads (Figure 4a). Phantoms are compatible with ultrasound imaging (Figure 4b and 4c) and have material properties mimicking abdominal aortic stiffnesses. Variations in the strain ratios in the anterior to posterior regions of the strain images demonstrate that the regions have varying material properties (Figure 5) and the independent mechanical testing on the samples quantify the exact values of their respective shear moduli.

\section{Discussion}

This paper presents a technique to manufacture tissue-mimicking phantoms for the use in testing elastography or elasticity imaging algorithms. The combined use of CAD and 3D printing allows for efficient design of aortic mimicking phantoms with complex geometries, beyond tubular phantoms, including aneurysmal bulges. The creation of the phantom is done in 4 steps; 1) design of the phantom geometry, 2) printing of the phantom mold parts, 3 ) mixing of the cryogel solutions which will ultimately mimic the ultrasonic characteristics and mechanical properties of the phantom vessels and 4) pouring/injecting of the cryogel solution into the mold, setting the PVA-c with freeze-thaw cycles and removal of the phantom from the mold. The use of CAD in the in the design of the molds obtained in Step 1 allows for a simple means to precisely modify the geometry of the phantoms. Printing of the mold parts currently takes approximately 5-8 $\mathrm{h}$ depending on the size of the print and thus can easily be made for repeated modifications to the molds. 
In Step 3, the cryogel solutions are created to mimic the vessel, aneurysm and background tissue with the calcium carbonate particles mimicking the US scatter of the tissue. The cryogel solutions should be stirred prior to use if the calcium particles have settled out of the mixture. The exact concentration of the mixture of the cryogel will determine the final mechanical properties of the phantoms. Thus, it is important to create the independent samples of each of the solutions used in the phantom vessel and background. Although not part of the protocol here, independent measurements of the sample's elastic modulus should be obtained using uniaxial tension testing. Independent mechanical testing of the PVA-c samples for the $10 \%, 15 \%, 20 \%$ and $25 \%$ phantoms created in the representative results had measured shear modulus of $17.4 \pm 1.0 \mathrm{kPa}, 48.3 \pm$ $5.7 \mathrm{kPa}, 95.1 \pm 0.4 \mathrm{kPa}$ and $170.0 \pm 4.1 \mathrm{kPa}$, respectively.

Step 4 is the most critical step in creating these phantoms. Although the registrations pins are in place to keep the mold parts in their proper positions relative to the others, it is important to ensure that the mold parts do not separate during the molding process. Thus, the use of the clamps to hold the mold. The most important consideration of Step 4 is to minimize air bubbles trapped in the mold prior to the first freeze-thaw cycle. It is often useful to disassemble one side of the outer mold and inspect the phantom after the first freeze-thaw cycle to ensure it formed properly. This can save time wasted putting a "bad" phantom through additional cycles. Once the phantom has been removed from the mold entirely, it can be stored in water for several weeks with continued use.

The PVA-c phantoms developed in this work were created to specifically mimic the ultrasonic and material stiffness of aortic tissue. The use of polyvinyl alcohol cryogel allows for a wider range of possible mechanical stiffness, to better mimic the changing material properties of aortic tissue compared to more rubber like materials ${ }^{33,34}$. In addition, the use of hydrogel and investment casting better captures the acoustic properties of casted rubbers or directly 3D printed materials ${ }^{33,45}$. Some air bubbles can get trapped in our molds prior to the first freeze-thaw cycle. This can cause gaps in the phantom and lead to material weakness or acoustic artifacts. Thus, it is recommended to inspect phantoms out of the mold after the first freeze-thaw to determine if the process should be restarted. In addition, the authors have found that the inner mold can sometimes shift during the freezing of the aneurysmal portion of the phantoms. If this occurs, one modification of the above protocol would be to create a $3 \mathrm{D}$ printed, or otherwise designed, part to firmly hold the inner lumen mold to the anterior outer mold during the freezing of this section. The authors have found that using the posterior side of the outer mold and a $5 \mathrm{~mm}$ spacer between the posterior outer mold and the inner mold works well for this purpose.

The phantom developed here is ideal for studying the influence of changes in aneurysmal diameter, and luminal thickness or potentially the presence of thrombus in the tissue by editing the original CAD files. However, previous work has also shown that this manufacturing technique can be modified to produce patient-specific phantom geometries using computed tomography images and segmentation software, rather than CAD design, to create the 3D printed phantom molds ${ }^{44}$. The results shown here demonstrate that the algorithm was able to visualize the manufactured variations in the mechanical properties of the phantom sections. It should be noted that although these phantoms were used to test US-based imaging techniques, they are also compatible with magnetic resonance and computed tomography imaging systems and that they may also be used beyond the purpose of elasticity imaging, for a wide range of novel imaging techniques and modalities.

\section{Disclosures}

The authors have nothing to disclose.

\section{Acknowledgements}

This work was supported by the National Center for Advancing Translational Sciences of the National Institutes of Health through Award No. UL1 TR000042 and the National Institute of Biomedical Imaging and Bioengineering of the National Institutes of Health through Award No. R21 EB018432.

1. Taylor, S. M. The Juxtarenal Abdominal Aortic Aneurysm. Archives of Surgery. 129 (7), 734-734, (1994).

2. Nevitt, M. P., Ballard, D. J., \& Hallett, J. W. Prognosis of Abdominal Aortic Aneurysms. The New England Journal of Medicine. 321 (15), 1009-1014, (1989).

3. Crane, C. Arteriosclerotic Aneurysm of the Abdominal Aorta. The New England Journal of Medicine. 253 (22), 954-958, (1955).

4. Trial Investigators. Endovascular or open repair strategy for ruptured abdominal aortic aneurysm: 30-day outcomes from IMPROVE randomised trial. British Medical Journal. 348 f7661, (2014).

5. Robinson, W. P. et al. Endovascular repair of ruptured abdominal aortic aneurysms does not reduce later mortality compared with open repair. Journal of Vascular Surgery. 63 (3), 617-624, (2016).

6. Starnes, B. W. et al. Management of ruptured abdominal aortic aneurysm in the endovascular era. Journal of Vascular Surgery. 51 (1), 9-18, (2010).

7. Schermerhorn, M. L. et al. Endovascular vs. Open Repair of Abdominal Aortic Aneurysms in the Medicare Population. The New England Journal of Medicine. 358 (5), 464-474, (2008).

8. Darling, R. C. et al. - Autopsy study of unoperated abdominal aortic aneurysms. The case for early. Circulation. 56 II161-164, (1977).

9. Szilagyi, D. E., Smith, R. F., DeRusso, F. J., Elliott, J. P., \& Sherrin, F. W. Contribution of abdominal aortic aneurysmectomy to prolongation of life. Annals of Surgery. 164 (4), 678-699, (1966).

10. Skibba, A. A. et al. Reconsidering gender relative to risk of rupture in the contemporary management of abdominal aortic aneurysms. Journal of Vascular Surgery. 62 (6), 1429-1436, (2015).

11. Parkinson, F. et al. Rupture rates of untreated large abdominal aortic aneurysms in patients unfit for elective repair. Journal of Vascular Surgery. 61 (6), 1606-1612, (2015).

12. Grant, S. W. W. et al. Calculating when elective abdominal aortic aneurysm repair improves survival for individual patients: development of the Aneurysm Repair Decision Aid and economic evaluation. Health technology assessment (Winchester, England). 19 (32), 1-154, (2015). 
13. Lederle, F. a. et al. Rupture rate of large abdominal aortic aneurysms in patients refusing or unfit for elective repair. JAMA: the journal of the American Medical Association. 287 (22), 2968-2972, (2002).

14. Vorp, D. A., Raghavan, M. L. L., \& Webster, M. W. Mechanical wall stress in abdominal aortic aneurysm: Influence of diameter and asymmetry. Journal of Vascular Surgery. 27 (4), 632-639, (1998).

15. Vande Geest, J. P. et al. Towards a noninvasive method for determination of patient-specific wall strength distribution in abdominal aortic aneurysms. Annals of Biomedical Engineering. 34 (7), 1098-1106, (2006).

16. Stringfellow, M. M., Lawrence, P. F., \& Stringfellow, R. G. The influence of aorta-aneurysm geometry upon stress in the aneurysm wall. Journal of Surgical Research. 42 (4), 425-433, (1987).

17. Maier, A. et al. A comparison of diameter, wall stress, and rupture potential index for abdominal aortic aneurysm rupture risk prediction Annals of Biomedical Engineering. 38 (10), 3124-3134, (2010).

18. Raghavan, M. L. L., Vorp, D. A., Federle, M. P., Makaroun, M. S., \& Webster, M. W. Wall stress distribution on three-dimensionally reconstructed models of human abdominal aortic aneurysm. Journal of Vascular Surgery. 31 (4), 760-769, (2000).

19. Di Martino, E. S. et al. Biomechanical properties of ruptured versus electively repaired abdominal aortic aneurysm wall tissue. Journal of Vascular Surgery. 43 (3), 570-576, (2006).

20. Gasser, T. C., Ogden, R. W., \& Holzapfel, G. a. Hyperelastic modelling of arterial layers with distributed collagen fibre orientations. Journal of the Royal Society, Interface / the Royal Society. 3 (6), 15-35, (2006).

21. Ruddy, J. M., Jones, J. A., Spinale, F. G., \& Ikonomidis, J. S. Regional heterogeneity within the aorta: Relevance to aneurysm disease. The Journal of Thoracic and Cardiovascular Surgery. 136 (5), 1123-1130, (2008).

22. Raut, S. S., Chandra, S., Shum, J., \& Finol, E. A. The role of geometric and biomechanical factors in abdominal aortic aneurysm rupture risk assessment. Annals of Biomedical Engineering. 41 (7), 1459-1477, (2013).

23. Tavares Monteiro, J. A. et al. Histologic, histochemical, and biomechanical properties of fragments isolated from the anterior wall of abdominal aortic aneurysms. Journal of Vascular Surgery. 59 (5), (2014).

24. Vallabhaneni, S. R. et al. Heterogeneity of tensile strength and matrix metalloproteinase activity in the wall of abdominal aortic aneurysms. Journal of endovascular therapy: an official journal of the International Society of Endovascular Specialists. 11 (4), 494-502, (2004).

25. Zou, Y., \& Zhang, Y. Mechanical evaluation of decellularized porcine thoracic aorta. TheJournal of Surgical Research. 175 (2), 359-368, (2012).

26. Ophir, J. et al. Elastography: Imaging the elastic properties of soft tissues with ultrasound. Journal of Medical Ultrasonics. 29 (4), 155-171, (2002).

27. Lopata, R. G. P. et al. Performance evaluation of methods for two-dimensional displacement and strain estimation using ultrasound radio frequency data. Ultrasound in Medicine and Biology. 35 (5), 796-812, (2009).

28. Fromageau, J. et al. in Ultrasonics Symposium, 2005 IEEE. 257-260 (IEEE). (2005).

29. Lopata, R. G. P. et al. Vascular elastography: A validation study. Ultrasound in Medicine and Biology. 40 (8), 1882-1895, (2014).

30. Mascarenhas, E. J. S. et al. Assessment of mechanical properties of porcine aortas under physiological loading conditions using vascular elastography. Journal of the Mechanical Behavior of Biomedical Materials. 59, 185-196, (2016).

31. Brekken, R. et al. Strain estimation in abdominal aortic aneurysms from 2-D ultrasound. Ultrasound in Medicine and Biology. 32 (1), $33-42$, (2006).

32. Vonk, T., Nguyen, V., Schurink, G., van de Vosse, F., \& Lopata, R. in Ultrasonics Symposium (IUS), 2014 IEEE International. 9 -12 (IEEE). (2014).

33. Cloonan, A. J. et al. 3D-Printed Tissue-Mimicking Phantoms for Medical Imaging and Computational Validation Applications. 3D Printing and Additive Manufacturing. 1 (1), 14-23, (2014).

34. Doyle, B. J. et al. Experimental modelling of aortic aneurysms: novel applications of silicone rubbers. Medical Engineering and Physics. $\mathbf{3 1}$ (8), 1002-1012, (2009).

35. Zell, K., Sperl, J., Vogel, M., Niessner, R., \& Haisch, C. Acoustical properties of selected tissue phantom materials for ultrasound imaging. Physics in Medicine and Biology. 52 (20), N475, (2007).

36. Surry, K., Austin, H., Fenster, A., \& Peters, T. Poly (vinyl alcohol) cryogel phantoms for use in ultrasound and MR imaging. Physics in Medicine and Biology. 49 (24), 5529, (2004).

37. Chu, K. C., \& Rutt, B. K. Polyvinyl alcohol cryogel: An ideal phantom material for MR studies of arterial flow and elasticity. Magnetic Resonance in Medicine. 37 (2), 314-319, (1997)

38. Richards, M. S. et al. Investigating the impact of spatial priors on the performance of model-based IVUS elastography. Physics in Medicine and Biology. 56 (22), 7223-7246, (2011).

39. Raghavan, M. L. et al. Regional distribution of wall thickness and failure properties of human abdominal aortic aneurysm. Journal of Biomechanics. 39 (16), 3010-3016, (2006).

40. Farsad, M., Zeinali-Davarani, S., Choi, J., \& Baek, S. Computational Growth and Remodeling of Abdominal Aortic Aneurysms Constrained by the Spine. Journal of Biomechanical Engineering. (2015).

41. Kim, J., Peruski, B., Hunley, C., Kwon, S., \& Baek, S. Influence of surrounding tissues on biomechanics of aortic wall. International Journal of Experimental and Computational Biomechanics. 2 (2), 105-117, (2013).

42. Lillie, J. S. et al. Pulse Wave Velocity Prediction and Compliance Assessment in Elastic Arterial Segments. Cardiovascular Engineering and Technology. 6 (1), 49-58, (2015).

43. Varble, N. et al. In vitro hemodynamic model of the arm arteriovenous circulation to study hemodynamics of native arteriovenous fistula and the distal revascularization and interval ligation procedure. Journal of Vascular Surgery. 59 (5), 1410-1417, (2014).

44. Mix, D. S. et al. Detecting Regional Stiffness Changes in Aortic Aneurysmal Geometries Using Pressure-Normalized Strain. Ultrasound in Medicine and Biology. 43 (10), 2372-2394, (2017).

45. Browne, J., Ramnarine, K., Watson, A., \& Hoskins, P. Assessment of the acoustic properties of common tissue-mimicking test phantoms Ultrasound in Medicine and Biology. 29 (7), 1053-1060, (2003). 